\title{
Theory of polarized fluorescence and absorption in molecular complexes comprising two chromophores with non-parallel absorption and emission transition dipole moments
}

\author{
Andrey A. Demidov, David L. Andrews \\ School of Chemical Sciences, University of East Anglia, Norwich NR4 7TJ, UK
}

Received 20 November 1994; in final form 18 January 1995

\begin{abstract}
This work concerns theoretical determination of the degree of fluorescence polarization and absorption anisotropy in double-chromophore complexes with non-parallel absorption and emission transition dipole moments. Specifically, the theory addresses the case in which energy may incoherently transfer within each complex between chromophores with broad and overlapped spectra. Inside each complex the chromophores are rigidly positioned and oriented relative to each other, whilst the complexes themselves are randomly distributed and oriented in space. The cases of steady-state and of $\delta$-pulse excitation are both considered. In general the results obtained apply not only to the specifically considered case of energy migration but more widely, for example to systems exhibiting fast trans-cis isomerization, electron or proton transfer, etc., either alone or in conjunction with conventional energy transfer.
\end{abstract}

\section{Introduction}

This work concerns a theoretical determination of the degree of fluorescence polarization and absorption anisotropy in double-chromophore complexes. Specifically, the theory addresses the case in which energy may incoherently transfer between chromophores within each individual complex. Such complexes are widely found in biochemical preparations; see for example Refs. [1-6]. Systems of this kind have chromophores with broad and frequently overlapped spectra, both for absorption and fluorescence. These features complicate the analysis of polarization spectra, and until recently there has been no theory appropriate for this particular case.

The basic theory of polarization phenomena in molecular pairs with incoherently related absorption and emission transitions was first developed in the 1920s [7]. This theory considered the case where only donor molecules absorb light and only accept acceptor molecules fluoresce. Recent results from the femtosecond laser spectroscopy of molecular pairs has stimulated new theoretical investigations of the role of coherence in such polarization phenomena [8-10]. However, the background for a theory applicable to incoherent energy transfer in complexes with the spectral characteristics cited in the previous paragraph has only recently been established [11-15]. In our work we continue the theoretical development by eliminating certain limitations that previously applied. Those limitations included the assumption that each chromophore has parallel absorption and emission transition dipole moments. We intend to remove such limitations by considering a more general case, i.e. where the absorption and emission transitions could be non-parallel. In practice such a case can be found when the 
chromophores are excited to higher singlet states $\left(S_{0} \rightarrow S_{n}\right.$ absorption transition, $\left.n \geqslant 2\right)$, with emission occurring from the first excited singlet $\left(S_{1} \rightarrow S_{0}\right.$ emission transition). The internal conversion $S_{n} \rightarrow S_{1}$ is here assumed to be much faster than the rates of inter-chromophore energy exchange and intrinsic fluorescence decay.

The schematic structure of a double-chromophore complex is presented in Fig. 1. In this figure the unit vectors $\mathbf{1}_{\mathrm{a}}$ and $\mathbf{1}_{\mathrm{f}}$ represent the directions of the absorption and emission transition dipole moments of the first chromophore, while $\mathbf{2}_{\mathrm{a}}$ and $\mathbf{2}_{\mathrm{f}}$ denote those of the second. Note that the two chromophores may be positioned at different locations; the figure shows their mutual orientations. The unit vector $e$ represents the polarization of the incident light. In each individual complex both chromophores are positioned and oriented rigidly relative to each other. Both of them can absorb light and fluoresce, and there is incoherent exchange of energy between them, but there is no interaction between separate complexes. Our aim is to find formulae for the fluorescence polarization and absorption anisotropy of these complexes randomly positioned and oriented in space. The cases both of steady-state and of $\delta$-pulse excitation will be considered.

\section{Steady-state excitation}

We begin by noting the Cartesian components of the vectors as presented in Fig. $1 ; \boldsymbol{e}=\left\{\sin \theta_{e} \cos \varphi_{e}\right.$, $\left.\sin \theta_{e} \sin \varphi_{e}, \cos \theta_{e}\right\}$ and $i=\left\{\sin \theta_{i} \cos \varphi_{i}, \sin \theta_{i} \sin \varphi_{i}, \cos \theta_{i}\right\}, i=\mathbf{1}_{\mathrm{a}}, \mathbf{1}_{\mathrm{f}}, \mathbf{2}_{\mathrm{a}}$ and $\mathbf{2}_{\mathrm{f}}$. The probability for the first chromophore to absorb excitation energy is governed by the rate $A_{1}=C \sigma_{1}\left(\lambda_{\mathrm{a}}\right)\left(e \cdot \mathbf{1}_{\mathrm{a}}\right)^{2}$ and for the second chromophore $A_{2}=C \sigma_{2}\left(\lambda_{\mathrm{a}}\right)\left(e \cdot \mathbf{2}_{\mathrm{a}}\right)^{2}$. Here $\sigma_{1,2}\left(\lambda_{\mathrm{a}}\right)$ are the absorption cross sections of the first and second chromophores at wavelength $\lambda_{\mathrm{a}} ; C$ is a constant of proportionality that will disappear in the final results.

In any given molecular complex the absorbed energy will redistribute between the chromophores in accordance with the following equations:

$$
n_{1}=g_{11} A_{1}+g_{12} A_{2}, \quad n_{2}=g_{21} A_{1}+g_{22} A_{2},
$$

where $n_{1}$ and $n_{2}$ are the probabilities to find the first and second chromophores, respectively, in their excited states. The matrix $g_{i j}$ has the form

$$
\|\mathbf{g}\|=D^{-1}\left\|\begin{array}{cc}
\tau_{2}^{-1}+K_{21} & K_{21} \\
K_{12} & \tau_{1}^{-1}+K_{12}
\end{array}\right\|,
$$

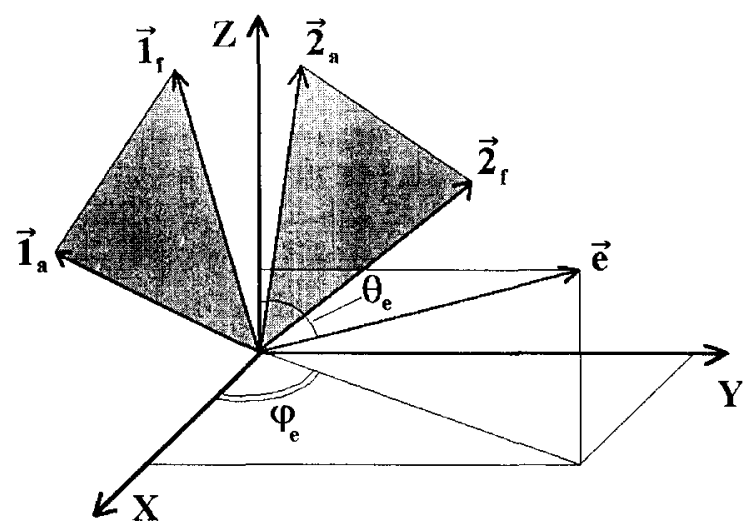

Fig. 1. Relative orientations of the absorption and emission transition dipole moments of one chromophore, $\mathbf{1}_{\mathrm{a}}$ and $\mathbf{1}_{\mathrm{f}}$ respectively, and another chromophore associated with $2_{\mathrm{a}}$ and $\mathbf{2}_{\mathrm{f}}$. The unit vector $e$ represents the polarization of the incident light; for simplicity the polarization vector of the emergent light is not shown. 
where the determinant $D$, given by $D=\left(\tau_{2}^{-1}+K_{21}\right)\left(\tau_{1}^{-1}+K_{12}\right)-K_{12} K_{21}$, is a constant that divides each element of the matrix on the right. Here $K_{12}$ and $K_{21}$ are the total rates of energy transfer, by any mechanism, from the first to the second chromophores and vice versa, $\tau_{1,2}$ are the intrinsic fluorescence lifetimes of the first and second chromophores.

The total intensity of emitted fluorescence in a system of double-chromophore complexes randomly distributed and oriented in space can be obtained by averaging Eqs. (1) over all possible mutual orientations of the incident light polarization vector $e$ with respect to a Cartesian frame attached to the molecular complex, as shown in Fig. 1. We express the fluorescence intensity ${ }^{1}$ at wavelength $\lambda_{\mathrm{f}}$ as photon flux per unit wavelength ${ }^{2}$ $\langle\Phi\rangle=\eta_{1} \tau_{1}^{-1} f_{1}\left(\lambda_{\mathrm{f}}\right)\left\langle n_{1}\right\rangle+\eta_{2} \tau_{2}^{-1} f_{2}\left(\lambda_{\mathrm{f}}\right)\left\langle n_{2}\right\rangle=\eta_{1} \tau_{1}^{-1} f_{1}\left(\lambda_{\mathrm{f}}\right)\left(g_{11}\left\langle A_{1}\right\rangle+g_{12}\left\langle A_{2}\right\rangle\right)+\eta_{2} \tau_{2}^{-1} f_{2}\left(\lambda_{\mathrm{f}}\right)\left(g_{21}\left\langle A_{1}\right\rangle+\right.$ $\left.g_{22}\left\langle A_{2}\right\rangle\right) ;$ and so finally

$$
\langle\Phi\rangle=\frac{1}{3} C\left\{\eta_{1} \tau_{1}^{-1} f_{1}\left(\lambda_{\mathrm{f}}\right)\left[g_{11} \sigma_{1}\left(\lambda_{\mathrm{a}}\right)+g_{12} \sigma_{2}\left(\lambda_{\mathrm{a}}\right)\right]+\eta_{2} \tau_{2}^{-1} f_{2}\left(\lambda_{\mathrm{f}}\right)\left[g_{21} \sigma_{1}\left(\lambda_{\mathrm{a}}\right)+g_{22} \sigma_{2}\left(\lambda_{\mathrm{a}}\right)\right]\right\} .
$$

Here $\eta_{1,2}$ are the fluorescence quantum yields of the 'free' chromophores, and $f_{1,2}\left(\lambda_{\mathrm{f}}\right)$ the fluorescence spectra normalised in the sense $\int f(\lambda) \mathrm{d} \lambda=1$.

The emitted fluorescence has a component parallel to the polarization of the incident light $\left\langle\Phi_{\|}\right\rangle=$ $\eta_{1} \tau_{1}^{-1} f_{1}\left(\lambda_{\mathrm{f}}\right)\left\langle n_{1}\left(e \cdot \mathbf{1}_{\mathrm{f}}\right)^{2}\right\rangle+\eta_{2} \tau_{2}^{-1} f_{2}\left(\lambda_{\mathrm{f}}\right)\left\langle n_{2}\left(e \cdot \mathbf{2}_{\mathrm{f}}\right)^{2}\right\rangle$, giving

$$
\begin{aligned}
\left\langle\Phi_{\|}\right\rangle= & \frac{1}{15} C\left\{g_{11}\left[\eta_{1} \tau_{1}^{-1} f_{1}\left(\lambda_{\mathrm{f}}\right)\right] \sigma_{1}\left(\lambda_{\mathrm{a}}\right)\left(1+2 \cos ^{2} \theta_{11}\right)+g_{12}\left[\eta_{1} \tau_{1}^{-1} f_{1}\left(\lambda_{\mathrm{f}}\right)\right] \sigma_{2}\left(\lambda_{\mathrm{a}}\right)\left(1+2 \cos ^{2} \theta_{21}\right)\right. \\
& \left.+g_{21}\left[\eta_{2} \tau_{2}^{-1} f_{2}\left(\lambda_{\mathrm{f}}\right)\right] \sigma_{1}\left(\lambda_{\mathrm{a}}\right)\left(1+2 \cos ^{2} \theta_{12}\right)+g_{22}\left[\eta_{2} \tau_{2}^{-1} f_{2}\left(\lambda_{\mathrm{f}}\right)\right] \sigma_{2}\left(\lambda_{\mathrm{a}}\right)\left(1+2 \cos ^{2} \theta_{22}\right)\right\} .
\end{aligned}
$$

In this equation $\theta_{i j}$ is the angle between transition dipole moments, $i$ denoting the chromophore responsible for absorption and $j$ fluorescence; for example $\theta_{12}$ is the angle between vectors $1_{\mathrm{a}}$ and $2_{\mathrm{f}}$ (see Fig. 1). By definition the degree of fluorescence polarization $P=\left(\left\langle\Phi_{\|}\right\rangle-\left\langle\Phi_{\perp}\right\rangle\right) /\left(\left\langle\Phi_{\|}\right\rangle+\left\langle\Phi_{\perp}\right\rangle\right)$, where $\left\langle\Phi_{\perp}\right\rangle$ is the fluorescence component perpendicular to the polarization of the incident light. The total fluorescence $\langle\Phi\rangle$ is equal to $\left\langle\Phi_{\|}\right\rangle+2\left\langle\Phi_{1}\right\rangle$. Thus, the degree of fluorescence polarization $P=(3 k-1) /(k+1)$, where $k=$ $\left\langle\Phi_{\|}\right\rangle(\langle\Phi\rangle)^{-1}$. Another frequently used parameter, the polarization anisotropy $r=\left(\left\langle\Phi_{\|}\right\rangle-\left\langle\Phi_{\perp}\right\rangle\right)(\langle\Phi\rangle)^{-1}$, obviously follows as $r=2 P /(3-P)=0.5(3 k-1)$.

We thus obtain the following formulae for the degree of fluorescence polarization and anisotropy,

$$
\begin{aligned}
& P=\frac{3 \cos ^{2} \theta_{11}-1+q_{1}\left(3 \cos ^{2} \theta_{21}-1\right)+q_{2}\left(3 \cos ^{2} \theta_{12}-1\right)+q_{3}\left(3 \cos ^{2} \theta_{22}-1\right)}{3+\cos ^{2} \theta_{11}+q_{1}\left(3+\cos ^{2} \theta_{21}\right)+q_{2}\left(3+\cos ^{2} \theta_{12}\right)+q_{3}\left(3+\cos ^{2} \theta_{22}\right)}, \\
& r=\frac{3 \cos ^{2} \theta_{11}-1+q_{1}\left(3 \cos ^{2} \theta_{21}-1\right)+q_{2}\left(3 \cos ^{2} \theta_{12}-1\right)+q_{3}\left(3 \cos ^{2} \theta_{22}-1\right)}{5\left(1+q_{1}+q_{2}+q_{3}\right)},
\end{aligned}
$$

where

$$
q_{1}=\alpha \frac{g_{12}}{g_{11}}=\alpha \frac{K_{21}}{K_{21}+\tau_{2}^{-1}}, \quad q_{2}=\gamma \frac{g_{21}}{g_{11}}=\gamma \frac{K_{12}}{K_{21}+\tau_{2}^{-1}}, \quad q_{3}=\alpha \gamma \frac{g_{22}}{g_{11}}=\alpha \gamma \frac{K_{12}+\tau_{1}^{-1}}{K_{21}+\tau_{2}^{-1}} .
$$

Here the parameters $\alpha$ and $\gamma$ are the relative efficiencies of light absorption ${ }^{3}, \alpha=\sigma_{2}\left(\lambda_{\mathrm{a}}\right) / \sigma_{1}\left(\lambda_{\mathrm{a}}\right)$, and fluorescence, $\gamma=\eta_{2} \tau_{1} f_{2}\left(\lambda_{\mathrm{f}}\right) / \eta_{1} \tau_{2} f_{1}\left(\lambda_{\mathrm{f}}\right)$, by the two chromophores.

Note that the rates $K_{12}$ and $K_{21}$ featured in (7) will in general be strongly and identically dependent on the distance between the chromophores, as determined by whatever specific mechanism they portray. For example

\footnotetext{
${ }^{1}$ Specifically, the spectral radiant energy density.

${ }^{2}$ Angular brackets here denote orientationally averaged values.

${ }^{3}$ In experiments the more commonly used parameters are molar extinction coefficients $\epsilon$, measured in mol $\mathrm{m}^{-2}$ units. In terms of these parameters $\alpha=\epsilon_{2}\left(\lambda_{\mathrm{a}}\right) / \epsilon_{1}\left(\lambda_{\mathrm{a}}\right)$.
} 
the radiative mechanism carries an inverse square dependence, the shorter-range Förster mechanism an inverse sixth power, and in general both are present together with an inverse fourth power term established by the unified theory of radiative and radiationless energy transfer [16,17]. Again, at still shorter distances, the exchange mechanism is characterized by an exponential range dependence. However in each case $K_{12}$ and $K_{21}$ factorize into orientation- and distance-dependent parts. Hence the $q$ parameters are distance-independent, provided $\tau_{1}$ and $\tau_{2}$ are sufficiently large. In contrast to our case, where the relative positions and orientations of the chromophores are fixed, there is of course a highly significant range-dependence of the polarization parameters in the case where the molecules are spatially independent and randomly oriented [17].

Formulae (5)-(7) have been derived for the case of fluorescence polarization, but the same formulae can be applied to the determination of absorption anisotropy in steady-state pump-probe measurements. Here we are considering experiments in which relative measurements are made of the probe signal absorption at a wavelength $\lambda_{\mathrm{p}}$ both in the presence and absence of pumping at another wavelength $\lambda_{\mathrm{a}}$; the difference, commonly known as the absorption recovery, is denoted by $\Delta A$. Despite the current consideration of steady-state excitation, the theory to describe such differential absorption as well as fluorescence can for example be applied under quasi steady-state conditions, such as where the energy transfer and fluorescence dynamics operate over a timescale much shorter than the duration of the excitation pulses. In this case we have $\alpha=\sigma_{2}\left(\lambda_{\mathrm{a}}\right) / \sigma_{1}\left(\lambda_{\mathrm{a}}\right)$ and $\gamma=\sigma_{2}\left(\lambda_{\mathrm{p}}\right) / \sigma_{1}\left(\lambda_{\mathrm{p}}\right)$. The anisotropy of absorption recovery, $r=\left(\Delta A_{\|}-\Delta A_{\perp}\right) /\left(\Delta A_{\|}\right.$ $+2 \Delta A_{\perp}$ ), can be calculated from (6) using the latter values for $\alpha$ and $\gamma$. In such pump-probe measurements the indices of the angles $\theta_{i j}$ have to be interpreted such that $i$ denotes the absorption transitions for the pump signal, and $j$ the absorption transitions for the probe signal. For example $\theta_{11}$ relates to pumping and probing transitions of the first type of chromophores while $\theta_{12}$ refers to pumping of the first chromophore type and probing of the second.

It is easily shown that in the degenerate case where $\theta_{11}=\theta_{22}=0$ and $\theta_{12}=\theta_{21}=\theta$, i.e. where each chromophore has parallel absorption and emission transition dipole moments, the fluorescence polarization degree has the following form:

$$
P=\frac{3 \cos ^{2} \theta-1+2 B}{3+\cos ^{2} \theta+4 B}, \quad B=\frac{\tau_{2}^{-1}+K_{21}+\alpha \gamma\left(\tau_{1}^{-1}+K_{12}\right)}{\alpha K_{21}+\gamma K_{12}} .
$$

This special case of the more general theory was previously treated in Ref. [12].

We can also consider another type of limiting behaviour, where excitation relaxation via intra-chromophore processes is much faster than inter-chromophore energy transfer: $\tau_{1,2}^{-1} \gg K_{i j}$. In this case the formulae (5) and (6) will take the form

$$
\begin{aligned}
& P=\frac{3 \cos ^{2} \theta_{11}-1+\alpha \gamma\left(\tau_{2} / \tau_{1}\right)\left(3 \cos ^{2} \theta_{22}-1\right)}{3+\cos ^{2} \theta_{11}+\alpha \gamma\left(\tau_{2} / \tau_{1}\right)\left(3+\cos ^{2} \theta_{22}\right)}, \\
& r=\frac{3 \cos ^{2} \theta_{11}-1+\alpha \gamma\left(\tau_{2} / \tau_{1}\right)\left(3 \cos ^{2} \theta_{22}-1\right)}{5\left(1+\alpha \gamma \tau_{2} / \tau_{1}\right)} .
\end{aligned}
$$

In general this limit represents quasi-independent behaviour of excitations located on the chromophores of the first and second type, so that no equilibration of energy between the chromophores takes place during the lifetime of the excitation. Evidently the above results reduce to the expected $P=0.5$ and $r=0.4$ when $\theta_{11}=\theta_{22}=0$.

Before concluding this section, it is worth noting that Eq. (6) affords an opportunity to correct a common misconception concerning the general principle of polarization anisotropy additivity. Although it is correct to assume that the polarization anisotropy of a whole system, as reflected by the parameter $r$, is a simple sum of the anisotropies of its constituent subsystems if the latter are uncorrelated, this is a principle that is not applicable to cases where there is orientational correlation within each unit, (see Fig. 1), as discussed here. 


\section{Pulsed excitation}

In the case of $\delta$-pulse excitation the balance of energy between the chromophores is described by the following system of equations:

$$
\frac{\mathrm{d} n_{1}}{\mathrm{~d} t}=-\left(\tau_{1}^{-1}+K_{12}\right) n_{1}+K_{21} n_{2}, \quad \frac{\mathrm{d} n_{2}}{\mathrm{~d} t}=K_{12} n_{1}-\left(\tau_{2}^{-1}+K_{21}\right) n_{2}
$$

with the initial conditions of excitation $n_{1}(0)=A_{1}$ and $n_{2}(0)=A_{2}$ (for the determination of $A_{1}$ and $A_{2}$ see above). The system (11) can be solved for the $n_{1,2}$,

$$
n_{1}(t)=g_{11}(t) A_{1}+g_{12}(t) A_{2}, \quad n_{2}(t)=g_{21}(t) A_{1}+g_{22}(t) A_{2}
$$

where the $g_{i j}(t)$ contain the same parameters as the former $g_{i j}$ as well as exponential functions of the times for the characteristic processes of energy equilibration $t_{1}$ and overall decay $t_{2}$,

$$
\begin{aligned}
t_{1}^{-1} & =0.5\left(\tau_{1}^{-1}+\tau_{2}^{-1}+K_{12}+K_{21}+\sqrt{\xi^{2}+4 K_{12} K_{21}}\right), \\
t_{2}^{-1} & =0.5\left(\tau_{1}^{-1}+\tau_{2}^{-1}+K_{12}+K_{21}-\sqrt{\xi^{2}+4 K_{12} K_{21}}\right), \\
\xi & =\tau_{2}^{-1}-\tau_{1}^{-1}+K_{21}-K_{12} .
\end{aligned}
$$

The system of Eq. (12) is analogous to the steady-state system (1) with elements $g_{i j}$ substituted by $g_{i j}(t)$. It follows that the final results will have the same form as Eqs. (5) and (6) with time dependence included in the $q$ parameters. Specifically, dynamical features of the fluorescence depolarization kinetics are manifest through the following expressions:

$$
\begin{aligned}
& P(t)=\frac{3 \cos ^{2} \theta_{11}-1+q_{1}(t)\left(3 \cos ^{2} \theta_{21}-1\right)+q_{2}(t)\left(3 \cos ^{2} \theta_{12}-1\right)+q_{3}(t)\left(3 \cos ^{2} \theta_{22}-1\right)}{3+\cos ^{2} \theta_{11}+q_{1}(t)\left(3+\cos ^{2} \theta_{21}\right)+q_{2}(t)\left(3+\cos ^{2} \theta_{12}\right)+q_{3}(t)\left(3+\cos ^{2} \theta_{22}\right)}, \\
& r(t)=\frac{3 \cos ^{2} \theta_{11}-1+q_{1}(t)\left(3 \cos ^{2} \theta_{21}-1\right)+q_{2}(t)\left(3 \cos ^{2} \theta_{12}-1\right)+q_{3}(t)\left(3 \cos ^{2} \theta_{22}-1\right)}{5\left[1+q_{1}(t)+q_{2}(t)+q_{3}(t)\right]}
\end{aligned}
$$

where

$$
\begin{aligned}
& q_{1}(t)=\alpha \frac{g_{12}(t)}{g_{11}(t)}=\alpha \frac{K_{21}[1-\exp (-\Omega t)]}{\left(K_{12}+\tau_{1}^{-1}-t_{2}^{-1}\right) \exp (-\Omega t)-\left(K_{12}+\tau_{1}^{-1}-t_{1}^{-1}\right)}, \\
& q_{2}(t)=\gamma \frac{g_{21}(t)}{g_{11}(t)}=\gamma \frac{K_{12}[1-\exp (-\Omega t)]}{\left(K_{12}+\tau_{1}^{-1}-t_{2}^{-1}\right) \exp (-\Omega t)-\left(K_{12}+\tau_{1}^{-1}-t_{1}^{-1}\right)}, \\
& q_{3}(t)=\alpha \gamma \frac{g_{22}(t)}{g_{11}(t)}=\alpha \gamma \frac{\left(K_{12}+\tau_{1}^{-1}-t_{2}^{-1}\right)-\left(K_{12}+\tau_{1}^{-1}-t_{1}^{-1}\right) \exp (-\Omega t)}{\left(K_{12}+\tau_{1}^{-1}-t_{2}^{-1}\right) \exp (-\Omega t)-\left(K_{12}+\tau_{1}^{-1}-t_{1}^{-1}\right)}, \\
& \Omega=t_{1}^{-1}-t_{2}^{-1} .
\end{aligned}
$$

Here the parameters $\alpha$ and $\gamma$ are the same as described above in connection with steady-state excitation.

Let us consider the particular case of $\theta_{11}=\theta_{22}=0$, i.e. when the absorption and emission transitions of individual chromophores are parallel, and consequently $\theta_{12}=\theta_{21}=\theta$, say. In this case Eq. (14), for example, can be simplified,

$$
P(t)=\frac{3 \cos ^{2} \theta-1+2 B(t)}{3+\cos ^{2} \theta+4 B(t)}, \quad B(t)=\frac{\xi(1-\alpha \gamma)+\left(t_{2}^{-1}-t_{1}^{-1}\right)(1+\alpha \gamma) \operatorname{coth}\left(\frac{1}{2} \Omega t\right)}{2\left(\alpha K_{21}+\gamma K_{12}\right)}
$$

and the result again reduces to a formula given in Ref. [12]. 
Finally we consider the limits of Eq. (14) as (a) $t \rightarrow 0$, (b) $t \rightarrow \infty$ and (c) $\tau_{1,2} \gg K_{i j}$.

(a) The initial value of the degree of polarization is given by

$$
\lim _{t \rightarrow 0}[P(t)]=\frac{3 \cos ^{2} \theta_{11}-1+\alpha \gamma\left(3 \cos ^{2} \theta_{22}-1\right)}{3+\cos ^{2} \theta_{11}+\alpha \gamma\left(3+\cos ^{2} \theta_{22}\right)},
$$

and in the particular case when $\theta_{11}=\theta_{22}=0$, we thus have $P(0)=0.5$.

(b) The formula for $P(t \rightarrow \infty)$ has the same form as Eq. (5) with the $q_{i}$ parameters given by

$$
q_{1}=\alpha, \quad q_{2}=\gamma \frac{K_{12}}{K_{21}} \quad \text { and } \quad q_{3}=\alpha \gamma \frac{K_{12}}{K_{21}},
$$

when $\tau_{1}=\tau_{2}$, or when $K_{i j} \gg \tau_{1,2}^{-1}$. The above results may be compared with the corresponding Eq. (7) for the steady-state case, in which the rates of fluorescence decay are manifest. Thus, we obtain the result that as $t \rightarrow \infty$ (or $t \gg \Omega^{-1}$ ) the degree of polarization equates to the result for steady-state excitation with $K_{i j} \gg \tau_{1,2}^{-1}$.

(c) In the case where $\tau_{1,2}^{-1} \gg K_{i j}$ the rates of intra-chromophore relaxation are much higher than the rates of excitation exchange between the chromophores. This limit yields quasi-independent behaviour of the chromophoric excitations and formula (14), for example, is simplified to

$$
P(t)=\frac{3 \cos ^{2} \theta_{11}-1+\alpha \gamma\left(3 \cos ^{2} \theta_{22}-1\right) \exp (-\Omega t)}{3+\cos ^{2} \theta_{11}+\alpha \gamma\left(3+\cos ^{2} \theta_{22}\right) \exp (-\Omega t)},
$$

with $\Omega$ reducing to $\tau_{2}^{-1}-\tau_{1}^{-1}$. The initial behaviour $(t \rightarrow 0)$ is obvious and directly follows from Eq. (20). It is interesting to note that the same constant value for the degree of polarization results, for any time $t$, if $\tau_{1}=\tau_{2}$,

$$
P(t)=\frac{3 \cos ^{2} \theta_{11}-1+\alpha \gamma\left(3 \cos ^{2} \theta_{22}-1\right)}{3+\cos ^{2} \theta_{11}+\alpha \gamma\left(3+\cos ^{2} \theta_{22}\right)} .
$$

The long-time limit $(t \rightarrow \infty)$ of $P$ is a very sensitive function of the relation between $\tau_{1}$ and $\tau_{2}$. If $\tau_{1}>\tau_{2}$, then $P(\infty)=\left(3 \cos ^{2} \theta_{11}-1\right) /\left(3+\cos ^{2} \theta_{11}\right)$, and if $\tau_{1}<\tau_{2}$, then $P(\infty)=\left(3 \cos ^{2} \theta_{22}-1\right) /\left(3+\cos ^{2} \theta_{22}\right)$.

\section{Discussion}

The theory of fluorescence polarization and absorption anisotropy introduced in our work focuses on a quantitative description of polarization phenomena in double-chromophore complexes having a rigid structure. We have considered the general case of non-parallel orientations for the transition moments within both chromophores, each of which can have broad and highly overlapped spectra. The major limitation involved in our calculations is consideration only of incoherent excitation, i.e. at any time the quantum of excitation is recognized as located on one or the other chromophore. The formulae derived allow calculation of the degree of fluorescence polarization (absorption anisotropy) on the basis of a known spatial structure for any given molecular complex and the spectroscopic parameters of its constituent chromophores. Equally, for example, measurement of the spectroscopic parameters, including polarization data, together with dynamical parameters such as energy transfer rates and fluorescence lifetimes, enables one to derive orientational information. The bichromophoric molecular system considered, with non-parallel absorption and fluorescence chromophoric transitions, has other polarization features that will become manifest with circularly polarized radiation ${ }^{4}$ [18]. Such features fall outside the scope of the present work, but represent a logical extension for the future.

\footnotetext{
${ }^{4}$ The chirality of a system with $\theta_{12}, \theta_{21} \neq 0$ can be manifest in circular differential excitation experiments. The magnitude of the differential signals will, however, depend on inter-chromophore separation.
} 
In the strategy of our calculation three major stages can be identified: (a) pumping of energy in the molecular complexes via light absorption by chromophores $\mathbf{1}_{\mathrm{a}}$ and $\mathbf{2}_{\mathrm{a}}$ of the first and second types, with the parameter $\alpha$ characterizing the relative efficiency of this process; (b) an intermediate stage associated with redistribution of the absorbed energy between the chromophores, to be discussed in more detail below; and (c) emission of fluorescence quanta by chromophoric transitions $\mathbf{1}_{\mathrm{f}}, \mathbf{2}_{\mathrm{f}}$, with a different relative efficiency of fluorescence $\gamma$.

The nature of the second stage in the above scheme can be several types. One such type has been considered in the main body of work, namely the case where the absorption and fluorescence are associated with different electronically excited states, for example where UV excitation initially populates $S_{n}$ states and the ensuing $S_{1} \rightarrow S_{0}$ fluorescence occurs in the visible or near-infrared range. Another possibility is where the initial excitation is directly to the $S_{1}$ state but, because of some fast conformational change, the orientations of the emission transitions $\mathbf{1}_{\mathrm{f}}, \mathbf{2}_{\mathrm{f}}$ differ from the absorption transitions $\mathbf{1}_{\mathrm{a}}, \mathbf{2}_{\mathrm{a}}$. Here the term 'fast' specifically means that the conformational change must be faster than the rate of energy transfer and/or intrinsic fluorescence $\left(\tau^{-1}\right)$.

Such a strategy of analysis leads us to the unexpected result that the formulae derived are not limited in their application to the case of energy migration, but have wider applicability. When we have double-chromophore complexes with fixed chromophore positions in the (a) and (c) stages, i.e. during the stages of energy pumping $\left(1_{\mathrm{a}}, \mathbf{2}_{\mathrm{a}}\right)$ and emission $\left(\mathbf{1}_{\mathrm{f}}, \mathbf{2}_{\mathrm{f}}\right)$, it is immaterial which mechanism causes transition between these two stages. If this transition can be described by any two-dimensional matrix $\|\mathbf{g}\|$ then our result will be valid by involving the appropriate elements: in such a case these elements will be determined by processes other than the energy migration. Processes like fast trans-cis isomerization, electron or proton transfer, etc., alone or in conjunction with conventional energy transfer, are thereby accommodated in our theory.

\section{Acknowledgement}

We are pleased to acknowledge funding by the Engineering and Physical Sciences Research Council.

\section{References}

[1] E. Gantt, Int. Rev. Cytol. 66 (1980) 45.

[2] A. Glazer, Biochim. Biophys. Acta 768 (1984) 29.

[3] A.D. Aleksic, M.F. Zloh and I. Juranic, J. Luminescence 59 (1994) 27.

[4] Z. Gryzczynski, T. Tenenholz and E. Bucci, Biophys. J. 63 (1992) 648.

[5] G. Marriott, W.R. Kirk, N. Johnsson and K. Weber, Biochemistry 29 (1990) 7004.

[6] M.G. Hyde, W. Mallawaarachchi and G.S. Beddard, Chem. Phys. Letters 187 (1991) 427.

[7] F. Perrin, Ann. Phys. (Paris) 12 (1929) 169.

[8] K. Wynne and R.M. Hochstrasser, Chem. Phys. 171 (1993) 179.

[9] R.S. Knox and D. Gülen, Photochem. Photobiol. 57 (1993) 40.

[10] H. van Amerongen and W.S. Struve, Methods Enzymol. 246 (1995) 259.

[11] A.A. Demidov, Appl. Optics 33 (1994) 6303.

[12] A.A. Demidov, J. Theoret. Biol. 170 (1994) 355.

[13] A.A. Demidov, Biophys. J. 67 (1994) 2184.

[14] P.A. Lyle and W.S. Struve, Photochem. Photobiol. 53 (1991) 359.

[15] D.L. Andrews and P. Allcock, in preparation.

[16] D.L. Andrews, Chem. Phys. 135 (1989) 195.

[17] D.L. Andrews and G. Juzeliūnas, J. Chem. Phys. 95 (1991) 5513.

[18] L.D. Barron, Molecular light scattering and optical activity (Cambridge Univ. Press, Cambridge 1982). 